\title{
WREATH PRODUCTS AND FORMATIONS OF GROUPS
}

\author{
DONALD B. PARKER ${ }^{1}$
}

1. Introduction. Let $A$ and $B$ be finite nontrivial groups and $A$ wr $B$ denote the wreath product of $A$ by $B$. In [1] Baumslag showed that $A$ wr $B$ is nilpotent if and only if $A$ and $B$ are both $p$-groups for the same prime $p$. In [2] Durbin proved that $A$ wr $B$ is supersolvable if and only if

(i) $A$ is nilpotent,

(ii) either $B$ is abelian or $B^{\prime}$ is a nontrivial p-group with $A$ a $p$-group for the same prime $p$, and

(iii) for each prime $q$ dividing $|A|, q \equiv 1(\bmod m)$, where $m$ is the exponent of $B / Q, Q$ being the Sylow $q$-subgroup of $B$.

Since both the properties nilpotency and supersolvability are examples of that class of group theoretical properties called locally defined formations [3], it is natural to ask more generally for a characterization of those groups $A$ and $B$ such that their wreath product lies in an arbitrary locally defined formation.

The main result of this paper is

THEOREM 2. Let $\left\{f_{p}\right\}$ be a collection of formations, one for each prime $p$ and let $f$ be the formation locally defined by the collection $\left\{f_{p}\right\}$. If $A$ and $B$ are nontrivial finite groups, then $A$ wr $B \in f$ if and only if the following conditions are satisfied:

(a) $p Y_{1}|A|$ and $p \nmid|B|$ if $f_{p}=\varnothing$,

(b) if $f_{p} \neq \varnothing$

(i) $B / F_{p}(B) \in f_{p}$ for each $p \nmid|A|$,

(ii) $B / O_{p}(B) \in f_{p}$ for each $p|| A \mid$ such that $F_{p}(A)=A$,

(iii) $\left[A / F_{p}(A)\right.$ wr $\left.B\right] \in f_{p}$ for each $p|| A \mid$ such that $F_{p}(A)<A$.

2. Notation and definitions. All groups considered are finite.

If $H$ is a subgroup of $A$ then $\Pi(H)=$ the direct product of $|B|$ copies of $H=$ the set of all functions from $B$ to $A$. $A$ wr $B=\prod(A) \cdot B$, the semidirect product of $\Pi(A)$ by $B$ with $\Pi(A)$ normal, where $f^{b}(x)=f\left(x b^{-1}\right)$ for $f \in \Pi(A)$ and $b \in B$. A finite group $H$ is said to be $p$-nilpotent if $H=P P^{*}$ where $P^{*}$ is a normal $p$-complement and $P$ is a Sylow $p$-group. $F_{p}(G)=$ the biggest $p$-nilpotent normal subgroup of $G . F(G)=$ the biggest nilpotent normal subgroup of $G$.

If $U$ is a set of primes then $O_{U}(G)=$ the biggest normal $U$-subgroup of $G$.

Received by the editors April 28, 1969.

1 These results form a part of the author's doctoral dissertation written at the Ohio State University in 1968 under the direction of Professor Wolfgang Kappe. 
We will write $O_{p}(G)$ for $O_{\{p\}}(G)$ whenever $p$ is a prime and $p^{\prime}$ will denote the set of primes distinct from $p$.

3. $F_{p}(A \operatorname{wr} B)$.

LemMA 1. $F_{p}(G)$ can be characterized by

(i) $O_{p^{\prime}}(G) \subseteq F_{p}(G)$ and

(ii) $O_{p}\left[G / O_{p^{\prime}}(G)\right]=F_{p}(G) / O_{p^{\prime}}(G)$.

Proof. $O_{p^{\prime}}(G)$ is trivially $p$-nilpotent since it is $p$-free and is a normal subgroup so $O_{p^{\prime}}(G) \subseteq F_{p}(G)$ and in fact since $F_{p}(G)=P P^{*}$ and $P^{*}$ is a characteristic subgroup we have $P^{*}=O_{p^{\prime}}(G)$. Thus $F_{p}(G) / O_{p^{\prime}}(G) \cong P$, hence is a $p$-group and is normal in $G / O_{p^{\prime}}(G)$ so $F_{p}(G) / O_{p^{\prime}}(G) \subseteq O_{p}\left[G / O_{p^{\prime}}(G)\right]$. If the containment were proper then the complete pre-image of $O_{p}\left[G / O_{p^{\prime}}(G)\right]$ would be a $p$-nilpotent normal subgroup of $G$ which properly contains $F_{p}(G)$.

LEMMA 2.

$$
O_{U}(A \operatorname{wr} B)= \begin{cases}\prod(A) \cdot O_{U}(B) & \text { if } A \text { is a } U \text {-group, } \\ \prod O_{U}(A) & \text { if } A \text { is not a } U \text {-group. }\end{cases}
$$

Proof. If $A$ is a $U$-group then so is the direct product $\prod(A)$ and hence $\Pi(A) \cdot O_{U}(B)$ is a normal $U$-group, therefore $\Pi(A) \cdot O_{U}(B)$ $\subseteq O_{U}(A$ wr $B)$.

If we denote by $B_{U}$ the group $B \cap O_{U}(A$ wr $B)$ then $B_{U}$ is a $U$-group, normal in $B$, so $B_{U} \subseteq O_{U}(B)$. But $O_{U}(A$ wr $B)=\prod(A) \cdot B_{U}$ $\subseteq \prod(A) \cdot O_{U}(B)$ therefore $O_{U}(A$ wr $B)=\prod(A) \cdot O_{U}(B)$.

If $A$ is not a $U$-group we note that in general if $N$ is a normal subgroup of a finite group $G$ and $O_{U}(G / N)=1$ then $O_{U}(N)=O_{U}(G)$. Since

$$
O_{U}\left[\Pi\left(O_{U}(A)\right)\right]=\prod\left(O_{U}(A)\right)
$$

it suffices to show $O_{U}\left[(A\right.$ wr $\left.B) / \prod\left(O_{U}(A)\right)\right]=1$.

Now if $N$ is a normal subgroup of $A$ then the group $(A / N)$ wr $B$ is isomorphic to the group $(A$ wr $B) / \Pi(N)$, so in particular letting $N=O_{U}(A)$ we see that it suffices to show $A / O_{U}(A)$ wr $B=1$. But the group $A^{*}=A / O_{U}(A)$ has the property that $O_{U}\left(A^{*}\right)=1$, so we need only show that $O_{U}\left(A^{*}\right)=1$ implies $O_{U}\left(A^{*}\right.$ wr $\left.B\right)=1$.

Suppose $O_{U}\left(A^{*}\right)=1$ and $O_{U}\left(A^{*}\right.$ wr $\left.B\right) \neq 1$. Since $O_{U}(M \times N)$ $=O_{U}(M) \times O_{U}(N)$ and $O_{U}(N)=O_{U}(G) \cap N$ for normal subgroups $M, \quad N$ of $G$ we have $1=\prod\left(O_{U}\left(A^{*}\right)\right)=O_{U} \prod\left(A^{*}\right)=\prod\left(A^{*}\right)$ $\cap O_{U}\left(A^{*}\right.$ wr $\left.B\right)$. Hence there is some $f b \in O_{U}\left(A^{*}\right.$ wr $\left.B\right)$ with $f \in \prod\left(A^{*}\right)$ and $1 \neq b \in B$. 
Let $1 \neq a \in A^{*}$ be arbitrary and define $g \in \Pi\left(A^{*}\right)$ by $g(1)=a$ and $g(x)=1$ for all $1 \neq x \in B$. Then $[g, f b] \in \prod\left(A^{*}\right) \cap O_{U}\left(A^{*}\right.$ wr $\left.B\right)=1$, in particular $[g, f b](1)=1$. But a direct evaluation gives

$$
\begin{aligned}
{[g, f b](1) } & =\left(g^{-1} g^{f b}\right)(1)=g^{-1}(1) \cdot\left(g^{f}\right)^{b}(1) \\
& =g^{-1}(1) \cdot g^{f}\left(b^{-1}\right)=g(1)^{-1} \cdot g\left(b^{-1}\right)^{f\left(b^{-1}\right)} \\
& =a^{-1} \cdot 1 \neq 1, \quad \text { a contradiction. }
\end{aligned}
$$

Thus $O_{U}\left(A^{*}\right.$ wr $\left.B\right)=1$.

Theorem 1. If $A$ and $B$ are nontrivial groups then

$$
\begin{aligned}
& F_{p}(A \operatorname{wr} B)=\prod(A) \cdot F_{p}(B) \quad \text { if } p \nmid|A| \\
& =\prod(A) \cdot O_{p}(B) \quad \text { if } p|| A \mid \text { and } F_{p}(A)=A \\
& =\prod\left(F_{p}(A)\right) \quad \text { if } p|| A \mid \text { and } F_{p}(A)<A \text {. }
\end{aligned}
$$

Proof. We use the characterization for $F_{p}(G)$ given in Lemma 1 . Case 1. Suppose $p \nmid|A|$. Then $A$ is a $p^{\prime}$-group hence $O_{p^{\prime}}(A$ wr $B)$ $=\prod(A) \cdot O_{p^{\prime}}(B)$ by Lemma 2 . Therefore $F_{p}(A$ wr $B) / O_{p^{\prime}}(A$ wr $B)$ $=F_{p}(A$ wr $B) / \prod(A) \cdot O_{p^{\prime}}(B)$ which is isomorphic to

$$
O_{p}\left[(A \text { wr } B) / \Pi(A) \cdot O_{p^{\prime}}(B)\right]
$$

by Lemma 1 . This in turn is isomorphic to $O_{p}\left[B / O_{p^{\prime}}(B)\right]$ because the groups inside the brackets [ ] are isomorphic. Finally $O_{p}\left[B / O_{p^{\prime}}(B)\right]$ is equal to $F_{p}(B) / O_{p^{\prime}}(B)$ again using the characterization for $F_{p}(G)$ given in Lemma 1 . But the complete pre-image of $F_{p}(B) / O_{p^{\prime}}(B)$ in $A$ wr $B / \Pi(A) \cdot O_{p^{\prime}}(B)$ under this isomorphism is

$$
\prod(A) \cdot F_{p}(B) / \Pi(A) \cdot O_{p^{\prime}}(B)
$$

therefore $F_{p}(A$ wr $B)=\prod(A) \cdot F_{p}(B)$.

Case 2. Suppose $p|| A \mid$. Then $A$ is not a $p^{\prime}$-group and Lemma 2 gives $O_{p}^{\prime}(A$ wr $B)=\prod\left(O_{p^{\prime}}(A)\right)$. Thus using Lemma 1

$F_{p}(A \operatorname{wr} B) / O_{p^{\prime}}(A$ wr $B)=O_{p}\left[(A \operatorname{wr} B) / \Pi\left(O_{p^{\prime}}(A)\right)\right] \cong O_{p}\left(A^{*} \operatorname{wr} B\right)$

where $A^{*}=A / O_{p^{\prime}}(A)$. To apply Lemma 2 we observe that by Lemma $1 A^{*}$ is a $p$-group if and only if $F_{p}(A)=A$. Hence $F_{p}(A$ wr $B)=$ $\prod(A) \cdot O_{p}(B)$ if $F_{p}(A)=A$. If $F_{p}(A)<A$, then

$F_{p}(A$ wr $B) / O_{p^{\prime}}(A$ wr $B)$

$$
\cong O_{p}\left(A^{*} \operatorname{wr} B\right)=\prod\left(O_{p}\left(A^{*}\right)\right)=\prod\left(F_{p}(A) / O_{p^{\prime}}(A)\right),
$$


which implies that $F_{p}(A$ wr $B)=\prod\left(F_{p}(A)\right)$.

The Fitting group $F(G)$ of a wreath product can be easily found using Theorem 1 and the fact that $F(G)=\cap F_{p}(G)$, the intersection taken over all primes $p$ ( $p$ dividing $|G|$ ). We state without proof the

Corollary. If $A$ and $B$ are nontrivial groups then

$$
\begin{aligned}
F(A \text { wr } B) & =\prod(A) \cdot O_{p}(B) & & \text { if } A \text { is a } p \text {-group, } \\
& =\prod(F(A)) & & \text { if more than one prime divides }|A| .
\end{aligned}
$$

4. Proof of Theorem 2. By definition [3], if $f$ is a formation locally defined by a set of formations $\left\{f_{p}\right\}$. Then $G \in f$ if and only if

(a) $p \nmid|G|$ if $f_{p}=\varnothing$,

(b) $G / F_{p}(G) \in f_{p}$ if $f_{p} \neq \varnothing$. Since $\mid A$ wr $B|=| A|| B|\cdot| B \mid$ applying Theorem 1 we see that $A$ wr $B \in f$ if and only if

(a) $p \nmid|A|, p \nmid|B|$ if $f_{p}=\varnothing$, and if $f_{p} \neq \varnothing$ then

(b) (i)* $(A$ wr $B) / \prod(A) \cdot F_{p}(B) \in f_{p}$ for each $p \nmid|A|$ $=A$.

(ii) ${ }^{*}(A$ wr $B) / \prod(A) \cdot O_{p} \in f_{p}$ for each $p|| A \mid$ such that $F_{p}(A)$

(iii)* $(A$ wr $B) / \Pi\left(F_{p}(A)\right) \in f_{p}$ for each $p|| A \mid$ such that $F_{p}(A)<A$.

By elementary isomorphism theorems for wreath products (i) ${ }^{*}$, (ii)* and (iii)* are equivalent to (i), (ii) and (iii) respectively.

5. Applications. If it is known which wreath products are contained in the $f_{p}$ which locally define $f$ then Theorem 2 can be used to characterize those $A$ wr $B \in f$ in terms of conditions on $A$ and $B$. Since the class of finite supersolvable groups can be described as the formation locally defined by $\left\{f_{p}\right\}$ where

$$
\begin{aligned}
f_{p}= & \text { the class of finite abelian groups of } \\
& \text { exponent dividing } p-1[3, \text { Beispiel 4.8] }
\end{aligned}
$$

condition (b) (iii) can never occur since no wreath product of nontrivial groups is ever abelian. That is to say $F_{p}(A)=A$ for all $p$, or equivalently $A$ is nilpotent. The characterization given by Durbin $[2]$ is then an easy consequence of Theorem 2.

Theorem 2 yields among others characterizations of $A$ wr $B \in f$ in terms of conditions on $A$ and $B$ for the following locally defined $f$ which are described in Huppert [4, VI $\S 7]$. We state the results without proof.

(a) $A$ wr $B$ is $p$-solvable of $p$-length at most 1 if and only if (i) $B / F_{p}(B)$ is $p$-free if $p \nmid|A|$, 
(ii) $B / O_{p}(B)$ is $p$-free if $p|| A \mid$ and $A$ is $p$-nilpotent,

(iii) $A / F_{p}(A)$ and $B$ are $p$-free if $p|| A \mid$ and $A$ is not $p$-nilpotent.

(b) If $\prec$ is an ordering of the primes then $A$ wr $B$ has an ordered $\prec-$ Sylow tower if and only if

(i) $B / F_{p}(B)$ is divisible only by primes $q$ with $p \prec q$ if $p \nmid|A|$,

(ii) $B / O_{p}(B)$ is divisible only by primes $q$ with $p \prec q$ if $p|| A \mid$ and $F_{p}(A)=A$,

(iii) $A / F_{p}(A)$ and $B$ are divisible only by primes $q$ with $p \prec q$ if $p|| A \mid$ and $F_{p}(A)<A$.

(c) $A$ wr $B$ is $p$-nilpotent if and only if

(i) $B$ is $p$-nilpotent if $p \nmid|A|$,

(ii) $B$ is a $p$-group and $A$ is $p$-nilpotent if $p|| A \mid$.

(d) If $f$ is the formation of those $p$-solvable groups whose $p$-chief factors have orders $p^{n_{1}}, \cdots, p^{n_{k}}$ such that 1.c.m. $\left(n_{1}, \cdots, n_{k}\right)=n$, then $A$ wr $B \in f$ if and only if either

(i) $p|| A \mid, A$ is $p$-nilpotent and $B / P$ is abelian of exponent dividing $p^{n}-1$ where $P$ is a $p$-Sylow group of $B$, or

(ii) $p \nmid|A|$ and $B / F_{p}(B)$ is abelian of exponent dividing $p^{n}-1$.

\section{REFERENCES}

1. G. Baumslag, Wreath products and p-groups, Proc. Cambridge Philos. Soc. 55 (1959), 224-231. MR 21\# 4179.

2. J. Durbin, Finite supersoliable wreath products, Proc. Amer. Math. Soc. 17 (1966), 215-218. MR 32\# 4192.

3. W. Gaschütz, Zur Theorie der endlichen auflösbaren Gruppen, Math. Z. 80 (1962/ 63) 300-305. MR 31\# 3505.

4. B. Huppert, Endliche Cruppen. I, Die Grundlehren der math. Wissenschaften, Band 134, Springer-Verlag, Berlin, 1967. MR 37\# 302.

University of Cincinnati 